\title{
Matrix Approach to The Direct Computation Method for The Solution of Fredholm Integro-Differential Equations of The Second Kind With Degenerate Kernels
}

\author{
Nathaniel Kamoh¹, Geoffrey Kumlengand², Joshua Sunday ${ }^{3}$ \\ 1,2,3 Department of Mathematics, University of Jos, Jos, Nigeria \\ Email: mahwash1477@gmail.com,gkumleng@gmail.com, \\ joshuasunday2000@yahoo.com
}

\begin{abstract}
In this paper, a matrix approach to the direct computation method for solving Fredholm IntegroDifferential Equations (FIDEs) of the second kind with degenerate kernels is presented. Our approach consists of reducing the problem to a set of linear algebraic equations by approximating the kernel with a finite sum of products and determining the unknown constants by the matrix approach. The proposed method is simple, efficient and accurate; it approximates the solutions exactly with the closed form solutions. The result of this research is the solution of the second type Fredholm integro-differential equation (FIDE) with a numerically accurate kernel degenerate. Some problems are considered using maple programme to illustrate the simplicity, efficiency and accuracy of the proposed method.
\end{abstract}

Keywords: Fredholm; Matrix; Direct Solution; Integro-Differential Equation; Integral

\section{INTRODUCTION}

The subject Integro-Differential Equations (IDEs) is one of the most important mathematical tools in both pure and applied mathematics. In recent year's mathematical modeling of real-life usually results in functional equations such as differential equations, integral and Integro-Differential Equations (IDEs) these equations play very important role in modern science and technology application such as the theory of signal processing, neutral networks, heat transfer, diffusion process, neutron diffusion and biological species. These equations can be classified into Fredholm equations and Volterra equations. The upper bound of the region for integral part of the Volterra type is a variable, while it is a fixed number for that of Fredholm type. More details and sources where these equations can be found are in the areas of Physics, biology, engineering and social sciences and have been extensively studied both at theoretical and practical level. Most integro-differential equations are usually very difficult to solve analytically and so accurate, acceptable and efficient numerical method is required to approximate the solution (see [1], [4], [5], [6], [13], [15] and [16]).

In recent times, extensive efforts have been devoted to the numerical methods of solution for Fredholm integro-differential equations by many researchers. [2] considered non-standard finite difference method for the numerical solution of linear Fredholm integro-differential equations. The method and the repeated composite 
trapezoidal quadrature method were used to transform the Fredholm integrodifferential equation into a system of non-linear algebraic equations and experiments on some linear model problems showed the simplicity and efficiency of the proposed method. The Wavelet method for the numerical solution of Fredholm integro-differential equation was used in [14]. [3] developed a finite difference hybrid method by a combination of power series and the shifted Legendre polynomial to solve Fredholm integro-differential equation. A new and efficient approach for the numerical solution of Fredholm integro-differential equations (FIDEs) of the second kind with an unbounded domain with degenerate kernel based on operational matrices with respect to generalized Laguerre polynomials (GLPs) was introduced in [8].The Adomian's decomposition method which is a well-known method for solving functional equations in recent times was used to solve linear Fredholm integro-differential equations by [9]. The result obtained gives more accurate approximation as compared to two other methods. [10], applied the Legendre polynomials for the solution of the linear Fredholm integro-differential-difference equation of high order and the results obtained by the developed technique were more accurate than the results reported for the Taylor and the wavelet Galerkin methods

The purpose of this paper is to solve Fredholm Integro-differential equation of the second kind with separable kernels by the approach of matrix method for the direct computation method, this approach is considered simple, accurate and easy to implement.

\section{METHODS}

Consider the standard form of the Fredholm integro-differential equation of the second kind given by

$$
\begin{aligned}
& \psi^{(n)}(\theta)=\omega(\theta)+\lambda \int_{a}^{b} k(\theta, \xi) \psi(\xi) d \xi \\
& \psi^{(k)}(0)=q_{k}, \quad 0 \leq k \leq n-1
\end{aligned}
$$

where $\psi^{(n)}(\theta)$ indicates the $n^{\text {th }}$ derivative of $\psi(\theta)$ with respect to $\theta$. Because (1)combines differential operator and the integral operator, then it is necessary to define initial conditions given in (2) for the determination of the particular solution $\psi(\theta)$ of (1). Suppose that we wish to determine the approximate solution of the theoretical solution $\psi(\theta)$ of problem (1) at the domain $[a, b]$. Let the separable or degenerate kernel $K(\theta, \xi)$ of (1) be approximated by $\sum_{k=1}^{n} \tau_{k}(\theta) \phi_{k}(\xi)$. Thus the integrodifferential equation (1) may be written as

$$
\psi^{(n)}(\theta)=\omega(\theta)+\lambda \int_{a}^{b} \sum_{k=1}^{n} \tau_{k}(\theta) \phi_{k}(\xi) \psi(\xi) d \xi,
$$

where $k(\theta, \xi)=\sum_{k=1}^{n} \tau_{k}(\theta) \phi_{k}(\xi)$ is a finite sum of products $\tau_{k}(\theta)$ and $\phi_{k}(\xi), \tau_{k}(\theta)$ is a function of $\theta$ only and $\phi_{k}(\xi)$ is a function of $\xi$ only.

Equation (3) can be rewritten as

$$
\psi^{(n)}(\theta)=\omega(\theta)+\lambda \sum_{k=1}^{n} \tau_{k}(\theta) \int_{a}^{b} \phi_{k}(\xi) \psi(\xi) d \xi
$$

Discretizing the integral part of (4) by letting $\mu_{k}=\int_{a}^{b} \phi_{k}(\xi) \psi(\xi) d \xi$, (4) becomes 


$$
\psi^{(n)}(\theta)=\omega(\theta)+\lambda \sum_{k=1}^{n} \tau_{k}(\theta) \mu_{k}
$$

Now, multiplying both sides of (5) by the integral operator $\chi^{-n}$ define by $\left(\chi^{-n}(\star)=\right.$ $\int(\star) d \theta$ ) with the application of the initial conditions (2), it follows that (5) can be written as

$$
\psi(\theta)-\sum_{i=1}^{n} \frac{\theta^{(i-1)}}{(i-1) !} \psi^{(i-1)}(0)=\chi^{-n}\left[\omega(\theta)+\lambda \sum_{k=1}^{n} \tau_{k}(\theta) \mu_{k}\right]
$$

Simplifying (6), we obtain

$$
\psi(\theta)-\sum_{i=1}^{n} \frac{\theta^{(i-1)}}{(i-1) !} \psi^{(i-1)}(0)=\omega^{*}(\theta)+\lambda \sum_{k=1}^{n} \tau_{k}^{*}(\theta) \mu_{k}
$$

Multiplying both sides of (7) by $\phi_{m}(\theta), m=1,2, \ldots, n$ and integrating from $a$ to $b$ over $\theta$ leads to a matrix equation that facilitates the determination of the $\mu_{k}$ 's in (5). Thus equation (7) becomes

$$
\begin{aligned}
& \int_{a}^{b} \psi(\theta) \phi_{m}(\theta) d \theta-\int_{a}^{b} \sum_{i=1}^{n} \frac{\theta^{(i-1)}}{(i-1) !} \psi^{(i-1)}(0) \phi_{m}(\theta) d \theta \\
&=\int_{a}^{b}\left(\omega^{*}(\theta)+\lambda \sum_{k=1}^{n} \tau_{k}^{*}(\theta) \mu_{k}\right) \phi_{m}(x) d \theta
\end{aligned}
$$

If we define $\mu_{m}, \omega_{m}, a_{m k}$ as $\int_{a}^{b} \psi(\theta) \phi_{m}(\theta) d \theta, \int_{a}^{b} \omega^{\star}(\theta) \phi_{m}(\theta) d \theta$ and $\int_{a}^{b} \tau^{*}{ }_{k}(\theta) \phi_{m}(\theta) d \theta$ respectively,then (8)can be written as

$$
\mu_{m}-\lambda \sum_{k=1}^{n} \mu_{k} a_{m k}=\omega_{m}, \quad m=1,2, \ldots, n
$$

Equation (9) gives a nonhomogeneous system of $n$ linear equations in $\mu_{1}, \mu_{2}, \mu_{3}, \ldots, \mu_{n}$ unknown. $\omega_{m}$ and $\tau_{m k}$ are known since $\phi_{m}(\theta), \omega^{\star}(\theta)$ and $\tau^{*}{ }_{k}(\theta)$ are all given.

Putting (9) in matrix equation form, it follows that

$$
\left(I-\lambda \sum_{k=1}^{n} a_{m k}\right) \mu_{k}=\omega_{m}
$$

where

$$
\mu_{k}=\left[\begin{array}{c}
\mu_{1} \\
\vdots \\
\mu_{n}
\end{array}\right], \quad \omega_{m}=\left[\begin{array}{c}
\omega_{1} \\
\vdots \\
\omega_{n}
\end{array}\right], \quad a_{m k}=\left[\begin{array}{ccc}
a_{11} & \cdots & a_{1 n} \\
\vdots & \ddots & \vdots \\
a_{n 1} & \cdots & a_{n n}
\end{array}\right]
$$

Equation (10)can be used to determine the unknown $\mu_{1}, \mu_{2}, \mu_{3}, \ldots, \mu_{n}$ which are then substituted into $\psi(\theta)=\omega^{*}(\theta)+\sum_{k=1}^{n} \tau_{k}^{*}(\theta) \mu_{k}$ for the particular solution of (1)

\section{RESULTS AND DISCUSSION}

We now apply the method presented in this paper to solved four model problems to illustrate the above mentioned approach and demonstrate its computational accuracy. This method differs from the direct computation method since the unknown constants are determined at once by the introduced matrix equation 


\section{Problem1}

Consider the linear Fredholm integro-differential equation given in [1]

$$
\begin{gathered}
y^{\prime \prime \prime}(x)=5 \ln 2-3-x+4 \cosh x-\int_{0}^{\ln 2}(x-t) y(t) d t, y(0)=y^{\prime \prime}(0)=0, y^{\prime}(0)=4, \\
0 \leq \theta \leq \pi
\end{gathered}
$$

The analytical solution to the problem is $y(x)=4 \sinh (x)$

Let

$$
\begin{gathered}
k(\theta, \xi)=\sum_{k=1}^{3} \tau_{k}(\theta) \phi_{k}(\xi)=(\theta-\xi) \\
\tau_{1}(\theta)=\theta, \tau_{2}(\theta)=-1, \phi_{1}(\xi)=1, \phi_{2}(\xi)=\xi
\end{gathered}
$$

Let the required solution be given as

$$
\psi(\theta)=\omega^{*}(\theta)+\sum_{k=1}^{3} \tau_{k}^{*}(\theta) \mu_{k}
$$

Multiplying the given FIDE through with the integral operator $\chi^{-3}$ to obtain

or

$$
\psi(\theta)-\sum_{i=1}^{3} \frac{\theta^{(i-1)}}{(i-1) !} \psi^{(i-1)}(0)=\omega^{*}(\theta)+\sum_{k=1}^{3} \tau_{k}^{*}(\theta) \mu_{k}
$$

$\psi(\theta)-\sum_{i=1}^{3} \frac{\theta^{(i-1)}}{(i-1) !} \psi^{(i-1)}(0)=2 e^{\theta}+\frac{5}{6} \theta^{3} \ln 2-\frac{1}{2} \theta^{3}-\frac{1}{24} \theta^{4}-\frac{2}{e^{\theta}}-\left(\frac{1}{24} \theta^{4} \mu_{1}-\frac{1}{6} \theta^{3} \mu_{2}\right)$

where

$$
\begin{aligned}
& \omega_{1}=\frac{1}{5} \ln 2^{5}-\frac{1}{8} \ln 2^{4}+1, \quad \omega_{2}=5 \ln 2-\frac{1}{10} \ln 2^{5}+\frac{23}{144} \ln 2^{6}-3 \\
& a_{11}=\frac{1}{120} \ln 2^{5}, a_{12}=-\frac{1}{24} \ln 2^{4}, a_{21}=\frac{1}{120} \ln 2^{6}, a_{22}=-\frac{1}{30} \ln 2^{5}
\end{aligned}
$$

Using (10) to solve for $\mu_{k}, k=1,2$, we have $\mu_{1}=1, \mu_{2}=5 \ln 2-3$ substituting in

$$
\psi(\theta)=2 e^{\theta}+\frac{5}{6} \theta^{3} \ln 2-\frac{1}{2} \theta^{3}-\frac{1}{24} \theta^{4}-\frac{2}{e^{\theta}}-\left(\frac{1}{24} \theta^{4} \mu_{1}-\frac{1}{6} \theta^{3} \mu_{2}\right)
$$

we have $\psi(\theta)=4 \sinh (\theta)$ as the exact solution. Table 1 below reveals the performance of the proposed method for problems 1

Table 1: The performance results of the proposed method for problem 1

\begin{tabular}{llll}
\hline values $(x=\theta)$ & Exact solution & Proposed Method & Absolute error \\
\hline 0.00 & 0.000000000 & 0.000000000 & $0.00 \mathrm{E}+00$ \\
0.10 & 0.400667000 & 0.400667000 & $0.00 \mathrm{E}+00$ \\
0.20 & 0.805344010 & 0.805344010 & $0.00 \mathrm{E}+00$ \\
0.30 & 1.218081174 & 1.218081174 & $0.00 \mathrm{E}+00$ \\
0.40 & 1.643009303 & 1.643009303 & $0.00 \mathrm{E}+00$ \\
0.50 & 2.084381222 & 2.084381222 & $0.00 \mathrm{E}+00$ \\
0.60 & 2.546614328 & 2.546614328 & $0.00 \mathrm{E}+00$ \\
0.70 & 3.034334807 & 3.034334807 & $0.00 \mathrm{E}+00$ \\
0.80 & 3.552423929 & 3.552423929 & $0.00 \mathrm{E}+00$ \\
0.90 & 4.106066904 & 4.106066904 & $0.00 \mathrm{E}+00$ \\
1.00 & 4.700804776 & 4.700804776 & $0.00 \mathrm{E}+00$ \\
\hline
\end{tabular}




\section{Problem2}

Consider the linear Fredholm integro-differential equation in [1] given by

$$
\begin{gathered}
y^{\prime \prime \prime}(x)=e-2-x+e^{x}(3+x)+\int_{0}^{1}(x-t) y(t) d t, y(0)=0, y^{\prime}(0)=1, y^{\prime \prime}(0)=2, \\
0 \leq \theta \leq \pi
\end{gathered}
$$

The analytical solution to the problem is $y(x)=x e^{x}$

Let

$$
\begin{gathered}
k(\theta, \xi)=\sum_{k=1}^{3} \tau_{k}(\theta) \phi_{k}(\xi)=(\theta-\xi) \\
\tau_{1}(\theta)=\theta, \tau_{2}(\theta)=-1, \phi_{1}(\xi)=1, \phi_{2}(\xi)=\xi
\end{gathered}
$$

The required solution is given as

$$
\psi(\theta)=\omega^{*}(\theta)+\sum_{k=1}^{3} \tau_{k}^{*}(\theta) \mu_{k}
$$

Multiplying the given FIDE with the integral operator $\chi^{-3}$, we have

$$
\psi(\theta)-\sum_{i=1}^{3} \frac{\theta^{(i-1)}}{(i-1) !} \psi^{(i-1)}(0)=\omega^{*}(\theta)+\sum_{k=1}^{3} \tau_{k}^{*}(\theta) \mu_{k}
$$

or

$$
\psi(\theta)-\sum_{i=1}^{3} \frac{\theta^{(i-1)}}{(i-1) !} \psi^{(i-1)}(0)=\frac{1}{6} e \theta^{3}-\frac{1}{3} \theta^{3}-\frac{1}{24} \theta^{4}+\theta e^{\theta}+\left(\frac{1}{24} \theta^{4} \mu_{1}-\frac{1}{6} \theta^{3} \mu_{2}\right)
$$

where

$$
\begin{gathered}
\omega_{1}=\frac{109}{120}+\frac{1}{24} e, \quad \omega_{2}=-\frac{1493}{720}+\frac{31}{30} e \\
a_{11}=\frac{1}{120}, \quad a_{12}=-\frac{1}{24}, \quad a_{21}=\frac{1}{144}, \quad a_{22}=-\frac{1}{30}
\end{gathered}
$$

Using (10) to solve for $\mu_{k}, k=1,2$ and substituting into

we obtain $\psi(\theta)=\theta e^{\theta}$ giving the same result as the exact solution.

$$
\psi(\theta)=\frac{1}{6} e \theta^{3}-\frac{1}{3} \theta^{3}-\frac{1}{24} \theta^{4}+\theta e^{\theta}+\left(\frac{1}{24} \theta^{4} \mu_{1}-\frac{1}{6} \theta^{3} \mu_{2}\right)
$$

\section{Problem 3}

Consider the linear Fredholm integro-differential equation in [1] given as

$$
y^{\prime \prime}(x)=4 x-\sin (x)+\int_{-\frac{\pi}{2}}^{\frac{\pi}{2}}(x-t)^{2} y(t) d t, y(0)=0, y^{\prime}(0)=1,-\frac{\pi}{2} \leq \theta \leq \frac{\pi}{2}
$$

The analytical solution to the problem is $y(x)=\sin (x)$

Let

$$
\begin{gathered}
k(\theta, \xi)=\sum_{k=1}^{2} \tau_{k}(\theta) \phi_{k}(\xi)=(\theta-\xi)^{2} \\
\tau_{1}(\theta)=\theta^{2}, \tau_{2}(\theta)=-2 \theta, \tau_{3}(\theta)=1 \phi_{1}(\xi)=1, \phi_{2}(\xi)=\xi, \phi_{3}(\xi)=\xi^{2}
\end{gathered}
$$


The required solution is given as

$$
\psi(\theta)=\omega^{*}(\theta)+\sum_{k=1}^{2} \tau_{k}^{*}(\theta) \mu_{k}
$$

Multiplying the given FIDE with the integral operator $\chi^{-2}$, we have

or

$$
\psi(\theta)-\sum_{i=1}^{2} \frac{\theta^{(i-1)}}{(i-1) !} \psi^{(i-1)}(0)=\omega^{*}(\theta)+\sum_{k=1}^{3} \tau_{k}^{*}(\theta) \mu_{k}
$$

where

$$
\psi(\theta)-\sum_{i=1}^{2} \frac{\theta^{(i-1)}}{(i-1) !} \psi^{(i-1)}(0)=\sin \theta+\frac{2}{3} \theta^{3}+\left(\frac{1}{2} \theta^{2} \mu_{3}-\frac{1}{3} \theta^{3} \mu_{2}+\frac{1}{12} \theta^{4} \mu_{1}\right)
$$

$$
\begin{array}{rlrl}
\omega_{1}=0, & \omega_{2}=\frac{1}{12} \pi^{5}+2, & \omega_{2}=0 \\
a_{11}=\frac{1}{960} \pi^{5}, & a_{12}=0, & a_{13}=\frac{1}{24} \pi^{3} \\
a_{21}=0, & a_{22}=-\frac{1}{240} \pi^{5}, & a_{23}=0 \\
a_{31}=\frac{1}{5376} \pi^{7}, a_{32}=0, & a_{33}=\frac{1}{160} \pi^{5}
\end{array}
$$

Using (10) to solve for $\mu_{k}, k=1,2,3$ and substituting in

$$
\psi(\theta)=\sin \theta+\frac{2}{3} \theta^{3}+\left(\frac{1}{2} \theta^{2} \mu_{3}-\frac{1}{3} \theta^{3} \mu_{2}+\frac{1}{12} \theta^{4} \mu_{1}\right)
$$

gives $\psi(\theta)=\sin (\theta)$ having the same result as the exact solution.

\section{Problem 4}

Consider the linear Fredholm integro-differential equation given by

$$
\begin{gathered}
y^{(i v)}(x)=2 x-\pi+\sin (x)+\cos (x)-\int_{0}^{\frac{\pi}{2}}(x-2 t) y(t) d t, \quad y(0)=y^{\prime}(0)=1, \\
y^{\prime \prime}(0)=y^{\prime \prime \prime}(0)=-1, \quad-\frac{\pi}{2} \leq \theta \leq \frac{\pi}{2}
\end{gathered}
$$

The analytical solution to the problem is $y(x)=\sin (x)+\cos (x)$

Let

$$
k(\theta, \xi)=\sum_{k=1}^{4} \tau_{k}(\theta) \phi_{k}(\xi)=(\theta-2 \xi)
$$

$\tau_{1}(\theta)=\theta, \tau_{2}(\theta)=-2, \tau_{3}(\theta)=0, \tau_{4}(\theta)=0, \phi_{1}(\xi)=1, \phi_{2}(\xi)=\xi, \phi_{3}(\xi)=0, \phi_{4}(\xi)=0$

The required solution is given as

$$
\psi(\theta)=\omega^{*}(\theta)+\gamma \sum_{k=1}^{4} \tau_{k}^{*}(\theta) \mu_{k}
$$

Multiplying the given FIDE with the integral operator $\chi^{-4}$, we have

$$
\psi(\theta)-\sum_{i=1}^{4} \frac{\theta^{(i-1)}}{(i-1) !} \psi^{(i-1)}(0)=\omega^{*}(\theta)+\sum_{k=1}^{4} \tau_{k}^{*}(\theta) \mu_{k}
$$


or

$$
\psi(\theta)-\sum_{i=1}^{4} \frac{\theta^{(i-1)}}{(i-1) !} \psi^{(i-1)}(0)=\cos \theta+\sin \theta-\frac{1}{24} \pi \theta^{4}+\frac{1}{60} \theta^{5}+\left(\frac{1}{12} \theta^{4} \mu_{2}-\frac{1}{120} \theta^{5} \mu_{1}\right)
$$

where

$$
\begin{array}{cc}
\omega_{1}=2-\frac{1}{4608} \pi^{6}, & \omega_{2}=\frac{1}{2} \pi-\frac{29}{322560} \pi^{7} \\
a_{11}=-\frac{1}{46080} \pi^{6}, & a_{12}=\frac{1}{1920} \pi^{5} \\
a_{21}=-\frac{1}{107520} \pi^{7}, & a_{22}=\frac{1}{4608} \pi^{6}
\end{array}
$$

Using (10) to solve for $\mu_{k}, k=1,2$ and substituting in

$$
\psi(x)=\cos \theta+\sin \theta-\frac{1}{24} \pi \theta^{4}+\frac{1}{60} \theta^{5}+\left(\frac{1}{12} \theta^{4} \mu_{2}-\frac{1}{120} \theta^{5} \mu_{1}\right)
$$

we obtain $\psi(\theta)=\cos (\theta)+\sin (\theta)$ giving the same result with the exact solution.

\section{CONCLUSIONS}

This paper deals with the solution of linear Fredholm integro-differential equations of the second kind with separable kernels. Our approach was based on the matrix approach which reduces the Fredholm integro- differential equation into a set of linear algebraic equations for the determination of the unknown constants. The advantage of this method over the direct computation method is that the constants in this method are obtained at once instead of the successive substitution approach inherent in the direct computation method. The method was tested on some model problems from the literature; the results obtained by the technique developed were the same with the exact solution revealing the effectiveness of the proposed method.

\section{ACKNOWLEDGMENTS}

The authors express their sincere thanks to the referees for the careful and details reading of their earlier version of the paper and for the very helpful suggestions.

\section{REFERENCES}

[1] Abdul-Majid Wazwaz, Linear and Nonlinear Integral Equations Methods and Applications. Higher Education Press, Beijing, 2011

[2] Pandey, P. K., Numerical Solution of Linear Fredholm Integro-Differential Equations by Non-standard Finite Difference Method, Applications and Applied Mathematics, An International Journal(AAM): (10)2, pp.1019-1026, 2015

[3] Kamoh, N. M. and Kumleng, G. M., Developing a Finite Difference Hybrid Method for Solving Second Order Initial-Value Problems for the Volterra Type IntegroDifferential Equations. Songklanakarin Journal of Science and Technology SJST2018-0171.R1, 2018

[4] Kamoh, N. M., Aboiyar, T. and Kimbir, A. R, Continuous Multistep Methods for Volterra Integro-Differential Equations of the Second Order, Science World Journal 12(3): pp.11-14, 2017 
[5] Kamoh, N. M. and Aboiyar, T., "Continuous linear multistep method for the general solution of first order initial value problems for Volterra integrodifferential equations", Multidiscipline Modeling in Materials and Structures, Vol. 14 Issue: 5, pp.960-969, https://doi.org/10.1108/MMMS-12-2017-0149

[6] D. C. Sharma and M. C. Goyal, Integral Equations, PHI Private Learning Material Ltd, Delhi 110092. 2017

[7] Behrouz Raftari, Numerical Solutions of the Linear Volterra Integro-differential Equations: Homotopy Perturbation Method and Finite Difference Method, World Applied Sciences Journal (9): pp. 7-12, 2010

[8] Matinfar, M. and Riahifar, A., Numerical solution of Fredholm integral-differential equations on unbounded domain, Journal of Linear and Topological Algebra, 04(01):pp.43- 52, 2015

[9] Vahidi, A. R., Babolian, E., Cordshooli, Gh. A. and Azimzadeh, Z., Numerical Solution of Fredholm Integro-Differential Equation by Adomian's Decomposition Method. International Journal of Mathematics Analysis, 3(36): pp.1769 - 1773, 2009

[10] Saadatmandia, A. and Dehghan, B., Numerical solution of the higher-order linear Fredholm integro-differential-difference equation with variable coefficients. Computers and Mathematics with Applications (59): pp.2996-3004, 2012

[11] Nas, S., Yalcinbas, S. and Sezer, M., A Taylor polynomial approach for solving high order linear Fredholm integro-differential equations, International Journal of Mathematics Education Science Technology 31 (2):pp.213-225, 2000

[12] Sezer, M. and Gulsu, M., Polynomial solution of the most general linear FredholmVolterra integro differential-difference equations by means of Taylor collocation method, Applied Mathematics and Computation,(185): pp. 646-657, 2007

[13] Sezer, M. and Gulsu, M., A new polynomial approach for solving difference and Fredholm integro-difference equations with mixed argument, Applied Mathematics and Computation (171): pp.332-344, 2005

[14] Behiry, S. H. and Hashish, H., Wavelet methods for the numerical solution of Fredholm integro-differential equations, International Journal of Applied Mathematics 11 (1): pp. 27-35, 2002

[15] Wazwaz, A.M., A reliable algorithm for solving boundary value problems for higher-order integro-differential equations, Applied Mathematics and Computation (118): pp.327-342, 2001

[16] Hosseini, S.M. and Shahmorad, S., Numerical solution of a class of IntegroDifferential equations by the Tau Method with error estimation. Applied Mathematics and Computation (136):pp. 559-570, 2003 
The algorithm for the implementation of the given problems using the Maple programme is outline below:

with(Student[Calculus 1$]$ ) :

$n:=?$ :

$\lambda:=?$

$f:=f(x)$

$g:=(\lambda) \operatorname{sum}(\tau[\mathrm{i}] \cdot \mathrm{mu}[\mathrm{i}], i=1 . . n)$

$\tau[i]:=\tau[i](x), i=1 . . n$

$\phi[i]:=\phi[i](t), i=1 . . n$

$f^{\circledast}:=\operatorname{int}(\operatorname{int}(f, x), x):$

$g^{\circledast}:=\operatorname{int}(\operatorname{int}(\mathrm{g}, x), x):$

$f[j]:=\operatorname{int}\left(\phi[j] \cdot f^{\circledast}, x=a . . b\right), j=1 . . n:$

$A:=\operatorname{Matrix}([[\operatorname{seq}(\operatorname{int}(\phi[1] \cdot \operatorname{int}(\operatorname{int}(\operatorname{int}(\operatorname{int}(\tau[\mathrm{j}], x), x), x), x), x=\mathrm{a} . . \mathrm{b}), j=1 . . n)]$,

$[\operatorname{seq}(\operatorname{int}(\phi[2] \cdot \operatorname{int}(\operatorname{int}(\operatorname{int}(\operatorname{int}(\tau[\mathrm{j}], x), x), x), x), x=\mathrm{a} . . \mathrm{b}), j=1 . . n)], \ldots,[\operatorname{seq}(\operatorname{int}(\phi[n]$

$\cdot \operatorname{int}(\operatorname{int}(\operatorname{int}(\operatorname{int}(\tau[\mathrm{j}], x), x), x), x), x=\mathrm{a} . . \mathrm{b}), j=1 . . n)]])$

with(LinearAlgebra) :

$B:=\operatorname{Matrix}(n, n$, shape $=$ identity $)-(\lambda) A:$

$C:=\operatorname{simplify}($ MatrixInverse $(B))$

$F:=\operatorname{Matrix}(n, 1,[f[j], j=1 . . n])$

$\%:=$ MatrixVectorMultiply $(\mathrm{C}, F)$ :

$v:=\operatorname{simplify}(\%):$

$\operatorname{Vector}(n, 1,[\mathrm{mu}[j], \mathrm{j}=1 . . \mathrm{n}])=\operatorname{Matrix}(n, 1,[v])$

$y:=\operatorname{eval}\left(f^{\circledast}+g^{\circledast},\{m u[j]=?, j=1 . . n\}\right)$ 